\title{
Complicated traumatic injuries in human brain aging and neurodegenerative diseases; a review
}

\begin{abstract}
Severe traumatic brain injury (TBI) is a well-established risk factor for a variety of neurodegenerative diseases including Alzheimer's disease, Parkinson's disease, and amyotrophic lateral sclerosis (ALS).The present review analyzes the relationship between severe and complicated human head trauma, brain aging and neurodegenerative diseases. The brain injury induces the following neuropathological changes: Brain atrophy and accumulation of hyperphosphorylated tau, amyloid plaques acutely after a single severe TBI, neurofibrillary and glial tangles, dystrophic neurites, $43 \mathrm{kDa}$ TAR DNA-binding protein (TDP-43) neuronal and glial aggregates, microvasculopathy, myelinated axonopathy, neuroinflammation, and white matter degeneration, neuroinflammatory reaction, activation of microglial cells, and severe excitotoxicity during ischemia. Light and electron microscopic studies demonstrate status spongiousus of brain parenchyma, dark ischemic neuron and glial cells, degeneration of myelinated axons and inflammatory reaction, increased vesicular and vacuolar transendothelial transport, formation of transendothelial channels, closed and open interendothelial junctions, thickening and vacuolization of basement membrane, swollen and activated pericites participating in transcapillary exchange, glio-basal dissociation, and proliferation of connective fibers. Cortical capillaries show blood bran barrier breakdown, perivascular hemorrhages and the formation of vasogenic and cytotoxic brain edema. Presence of swollen glycogen rich- and glycogen depleted astrocytes, indicating mobilization of glycogen granules to supply ischemic neurons, ischemic and hydropic oligodendrocytes, degenerated myelinated axons, nerve cell death types, such as apotosis oncosis, autophagic and necrotic nerve cell types, beaded shape and vacuolated dendrites, synaptic plasticity, synaptic degeneration and synaptic junction disassembly. Nerve cell organelles show clear, dense and necrotic mitochondria, lacunar enlargement of rough endoplasmic reticulum and ribosome detachment, fragmentation of Golgi apparatus, and increased amount of lipofucsin granules in neurons and astrocytes. Age-related white matter changes influence the progression of various brain disorders, including ischemic and hemorrhagic stroke, Alzheimer's disease, and Parkinson's disease.
\end{abstract}

Keywords: neurons, neuroglial cells, brain trauma, brain aging, microscopy
Volume 9 Issue 4 - 2019

Orlando J Castejón

Universidad del Zulia,Venezuela

Correspondence: Orlando J Castejón, Faculty of Medicine, Universidad del Zulia, Apartado 526, Maracaibo 4003-A, Venezuela, Tel 58414621 8209, Email ocastejo@gmail.com

Received: May 06, 2017| Published: July 16, 2019
Abbreviations: TBI, traumatic brain injury; ALS, traumatic brain injury; TDP-43, TAR DNA-binding protein; PD, parkinson's disease; HD, huntington's disease; $\mathrm{AD}$, alzheimer's disease

\section{Introduction}

Electron microscopy studies of severe human TBI and aging brain offer the possibility to increase our understanding of brain aging mechanisms, neuroinflammation, and neurodegeneration following TBI. The present review analyzes the relationship between complicated head human trauma, brain aging and neurodegenerative diseases.

\section{Brain trauma as a risk for neurodegenerative diseases}

An exact connection between severe traumatic injuries and brain aging remains partially unknown until now. Severe traumatic brain injury (TBI) is a well-established risk factor for a variety of neurodegenerative diseases including Alzheimer's disease, Parkinson's disease, and amyotrophic lateral sclerosis (ALS). However, the pathologic phenotype of these post-traumatic neurodegenerations is largely unknown and there may be pathobiological differences between post-traumatic disease and the corresponding sporadic disorder. ${ }^{1}$ Head trauma is considered to be a risk factor for Alzheimer's disease, because a high prevalence of beta AP deposits has repeatedly been reported in patients who died within a few days following head injury. The density of beta AP diffuse deposits was linked only to aging and the presence of senile plaques. ${ }^{2}$ Irimia et al. ${ }^{3}$ emphasize the role of cerebral microhemorrhages due to traumatic brain injury and their effects on the aging human brain. According to these Authors, cerebral microbleeds (CMBs) are frequently associated with traumatic brain injury and have potential effects on cognitive and neural outcome as a function of age at injury.

Hanif et al. ${ }^{4}$ examined the age related outcome in acute subdural hematoma following traumatic head injury and concluded that the elderly are most predisposed to bleeding due to normal cerebral atrophy related to aging, stretching the bridging veins from the dura matter.

\section{Brain trauma and neuropathological changes on brain aging}

According to Bigler et al. ${ }^{5}$ age-related changes cause minimal temporal lobe gyral, hippocampal, temporal horn, and white matter atrophy. Brain trauma produced disproportionate white matter loss 
associated with increased temporal horn and sulcal CSF volumes; it caused substantial hippocampal atrophy, which was related to memory impairment. Gyral volume did not decrease, although it was related to memory performance. Miakotnykh et al. ${ }^{6}$ found predominance of vessels neurological symptoms in combination with cranial-cerebral trauma and ischemic stroke. Cerebral vessels pathology always predominates as regards cerebral traumatic pathology in old patients.

A systematic submicroscopic study have been reported by us in the last four decades on complicated traumatic brain injuries in young and elderly patients, using cortical biopsies obtained during neurosurgical treatment of patients with severe and complicated brain trauma.

\section{Increased vesicular and vacuolar transendothelial transport}

The cortical capillaries exhibit increased vesicular and vacuolar trasnendothelial transport (Figure 1). ${ }^{7,8}$ In addition we found closed and open endothelial junctions (Figure 2) ${ }^{9}{ }^{910}$ Some cortical capillaries show in severely edematous areas the formation of transendothelial channels. ${ }^{11}$ Cortical capillary showing deep invaginations of peripheral endothelial cytoplasmic zone (PEZ) extending to the vicinity of basement membrane (BM) (long arrows) and closed interendothelial junction (short arrows), suggesting parajunctional transitory transendothelial channel formations to increase transfer of proteinaceous edema fluid from capillary lumen toward the brain parenchyma as a pathogenetic mechanism of vasogenic brain edema. Thickening and vacuolization of basement membrane, and glio-basal dissociation, and proliferation of connective fibers are observed after repeated ischemic and hypoxic processes (Figure 3). ${ }^{12,13}$ The pericites embedded into the basement membrane show shuttle micropinocitotic vesicles indicating their participation on transcapillary transport (Figure 4). ${ }^{14,15}$

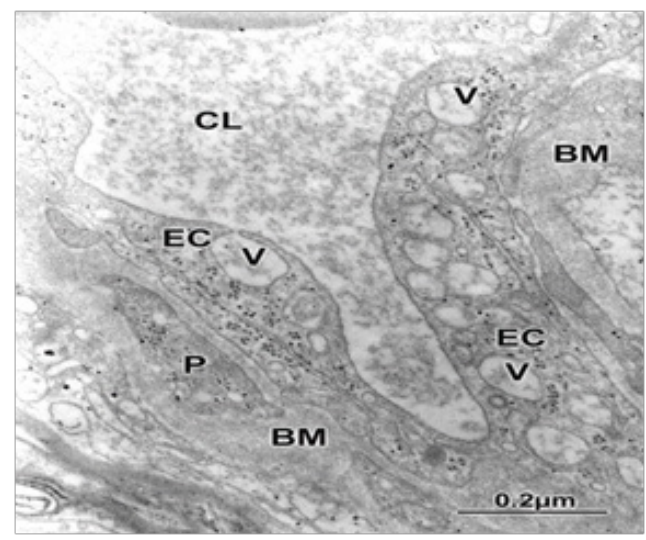

Figure I Brain trauma. Subdural hematoma. Cortical capillary showing increased vacuolar transport $(\mathrm{V})$ at the endothelial cell peripheral cytoplasm (EC), suggesting the genesis of vasogenic brain edema. Note the thickening basement membrane (BM) and the embedded pericite (P). The capillary lumen $(\mathrm{CL})$ exhibits the plasma serum proteinaceous granular substance.

\section{Brain traumatic injuries, nerve cell death and aging brain}

We found swollen and ischemic neurons and glial cells leading to nerve cell death types, such as apoptosis, oncosis, autophagic cell death leading to necrosis (Figure 5). ${ }^{16,17}$ Astrocyte exhibiting apoptotic cell death type showing apoptotic bodies in nucleus and cytoplasm (Figure 6). Morphopatological changes of dendrites ${ }^{17}$ and dendritic spines ${ }^{18,19}$ are observed. The dendrites show a beaded shape, vacuolization, edematous mitochondria and cytoskeletal disruption (Figure 7). Dendritic postynaptic spines appear swollen and contain an atrophic spine apparatus. They are observed making axospinodendritic synapses (Figure $8 \& 9$ ).

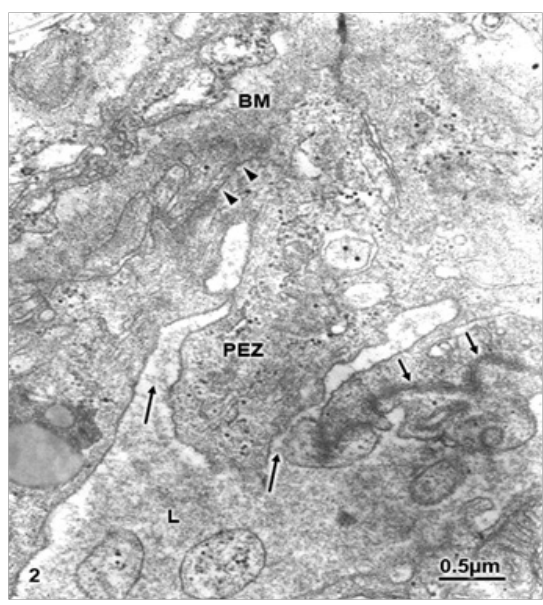

Figure 2 Brain trauma subdural hematoma.

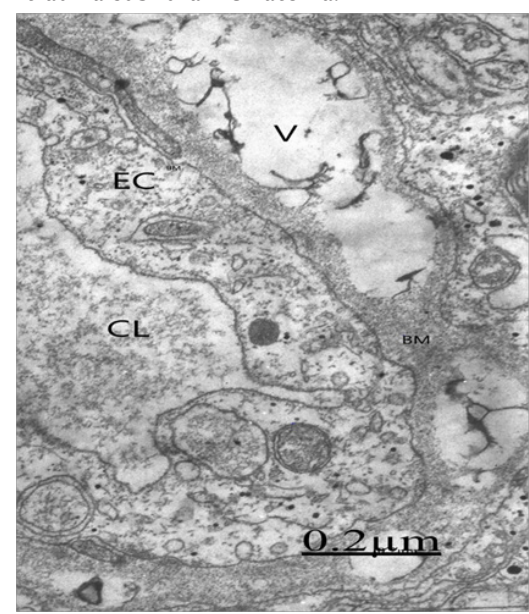

Figure 3 Brain trauma. Subdural hematoma. Severe brain edema showing thickening and vacuolization of basement membrane indicating complete blood brain barrier dysfunction.

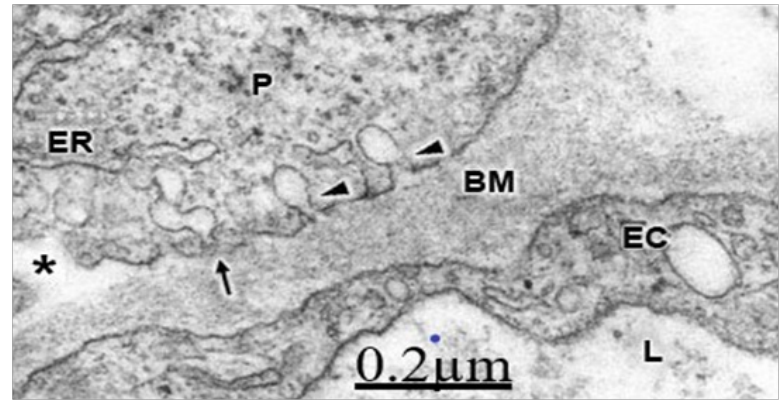

Figure 4 Brain trauma, subdural hematoma Severe brain edema depicting isolated (arrowheads) and shuttled micropinocytotic vesicles (arrow) discharging toward the swollen and vacuolated basement membrane (BM) The above findings suggest blood brain barrier breakdown and the genesis of vasogenic brain edema in complicated traumatic brain injuries. 


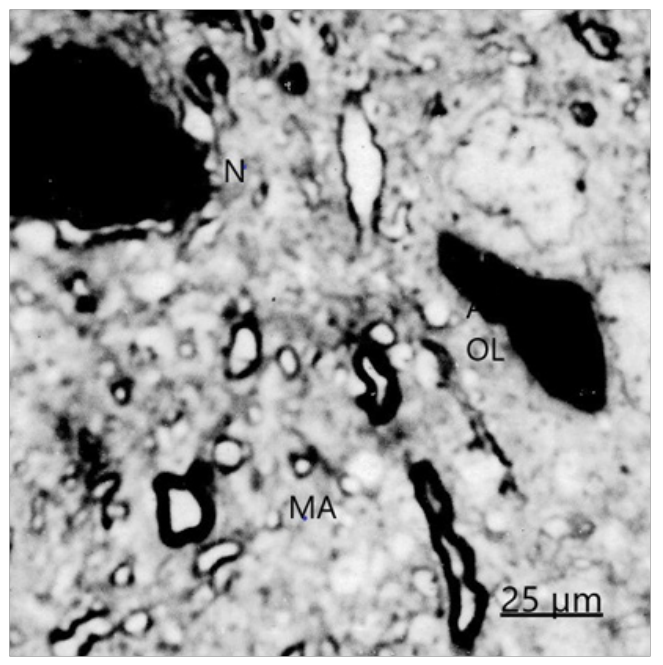

Figure 5 Brain trauma, subdural hematoma- Light microscopy section showing dark necrotic neuron $(N)$ and oligodendroglial cell $(O L)$ in the vicinity of an edematous astrocyte (A). Note the degenerated myelinated axons (MA).

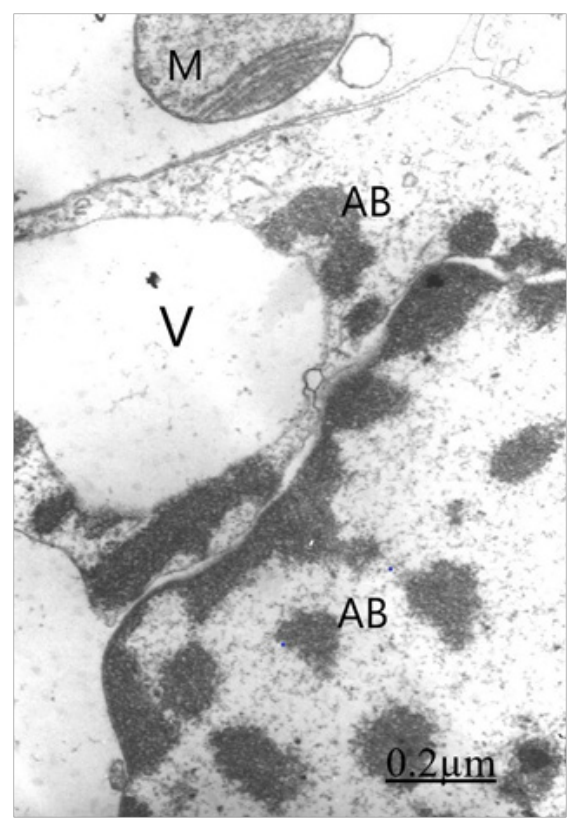

Figure 6 Brain trauma, hematoma subdural.Astrocyte cell showing apoptotic bodies $(A B)$ in nucleus $(N)$ and cytoplasm. A swollen and degenerated mitochondrion $(M)$ also is seen.

\section{Brain trauma, brain aging and neuroglial cell pathology}

We have reported glycogen rich-astrocyte and glycogen depletedastrocytes, ${ }^{20,21}$ which suggest anaerobic mobilization of glycogen stores in the human edematous cerebral cortex of elderly patients, which are rapidly depleted during anoxic-ischemic conditions. Such mobilization was earlier postulated by Philbin and Ransom. ${ }^{22}$

In addition, we found neurons and astrocytes containing heavy deposits of lipofucsin granules (Figure 10). Lipofucsin granules have been classically related with the aging process, and they are substantially increased with traumatic brain injury. ${ }^{23,24}$

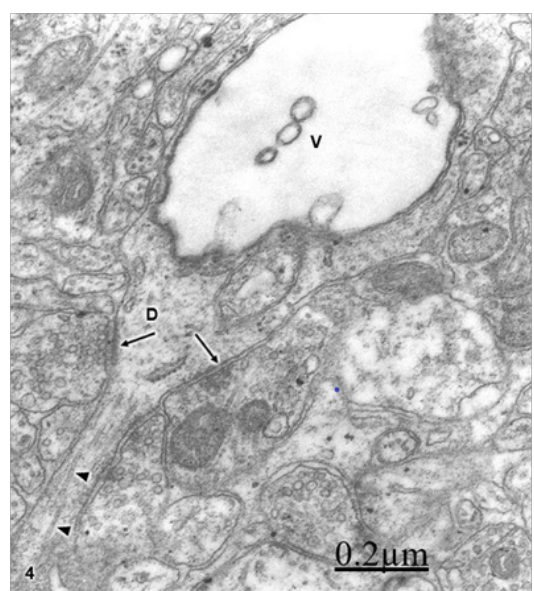

Figure 7 Brain trauma. Subdural hygroma. Swollen and vacuolated dendrite (V) exhibiting some axodendritic synapses (arrows). Some fragmented microtubules (arrowheads) also are distinguished.

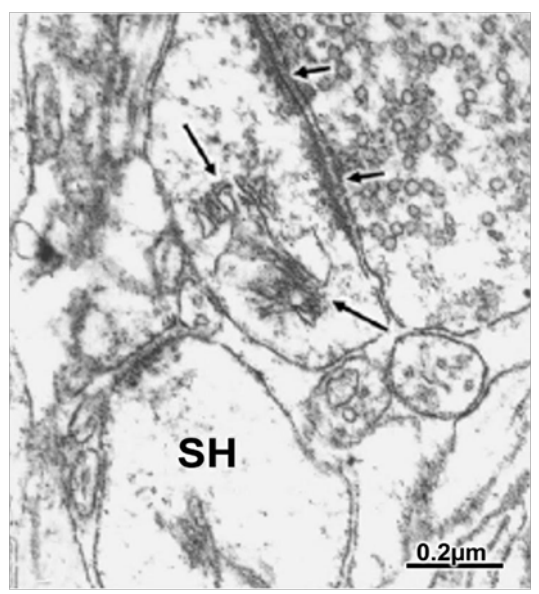

Figure 8 Brain trauma extradural hematoma. Postsynaptic spine containing an atrophic spine apparatus (long arrows) and establishing synaptic contact with a degenerated presynaptic ending with dispersed and aggregated synaptic vesicles. The short arrows indicate the preserved synaptic membrane complex.

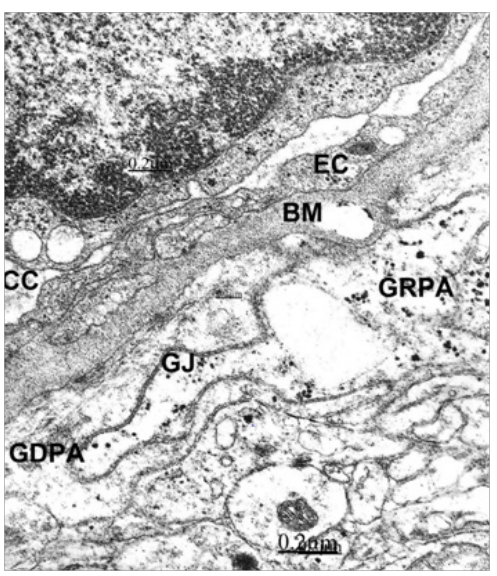

Figure 9 Brain traumas, extradural hematoma. Glycogen rich- (GRPA) and glycogen depleted- (GDPA) perivascular end-feet astrocytes (GR) separated by a gap junction (GJ) suggesting anaerobic mobilization of glycogen granules. They appear applied to the capillary vacuolated and thickened basement membrane outer surface. The endothelial cell (EC) and the collapsed capillary (CC) also are seen. 


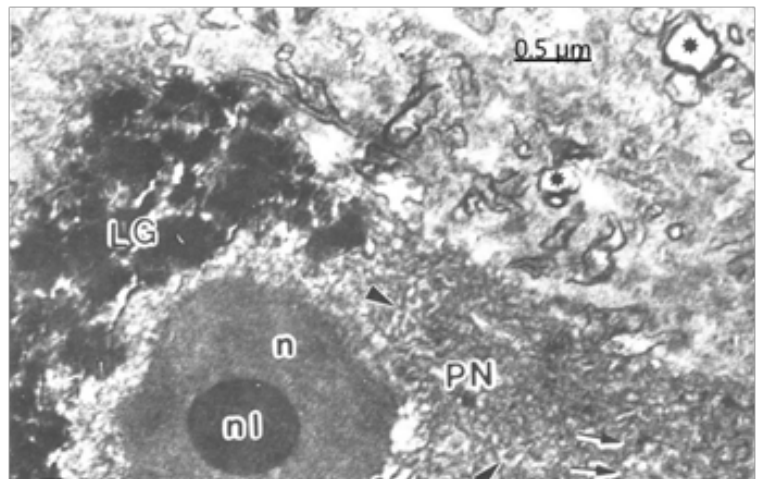

Figure 10 Brain trauma. Subdural hygroma. Scanning-transmission electron micrograph of a semithin plastic section of a pyramidal neuron (PN) showing the nucleus (n)and the round nucleolus ( $\mathrm{nl})$. Note the clustered deposit of cytoplasmic lipofucsin granules (LG).The arrowhead indicates cytomembranes.

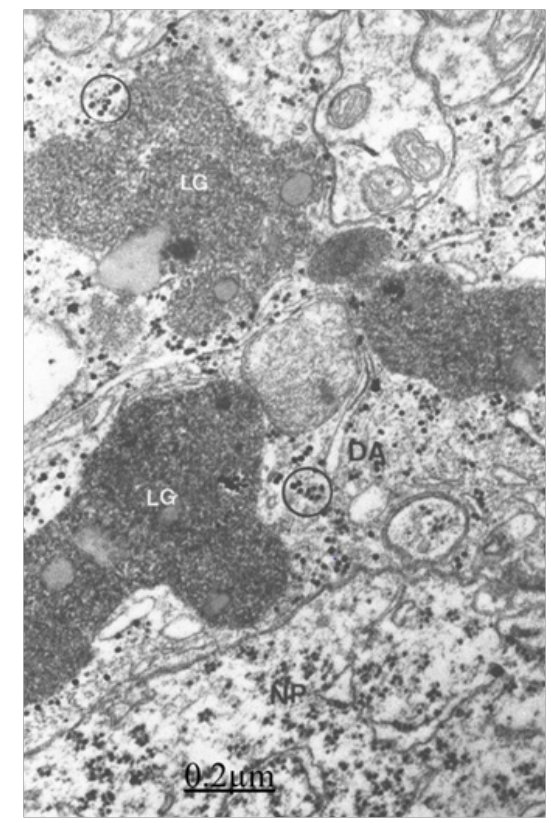

Figure I I Brain traumas, subdural hematoma. Non-pryramidal nerve cell (NP) surrounded by a satellite and peripheral dense astrocyte (DA) containing large lipofucsin granules (LG) and glycogen granules (circles).

Electron microscopy images of astrocytes at low magnification show nerve cells charged of numerous lipofucsin granules (Figure 11). Besides, we report edematous and ischemic oligodendrocytes (Figure 12). ${ }^{25,26}$

\section{Brain trauma, brain aging, synaptic plasticity and synaptic degeneration}

We have reported synaptic plasticity and synaptic degenerative changes of cortical axodendritic and axosomatic synapses (Figure 13). ${ }^{27,28}$ The synaptic degeneration is characterized by swollen of pre- and postsynaptic endings, coalescence presynaptic vesicles and filamentous hypertrophy (Figures 14\&15). ${ }^{28}$

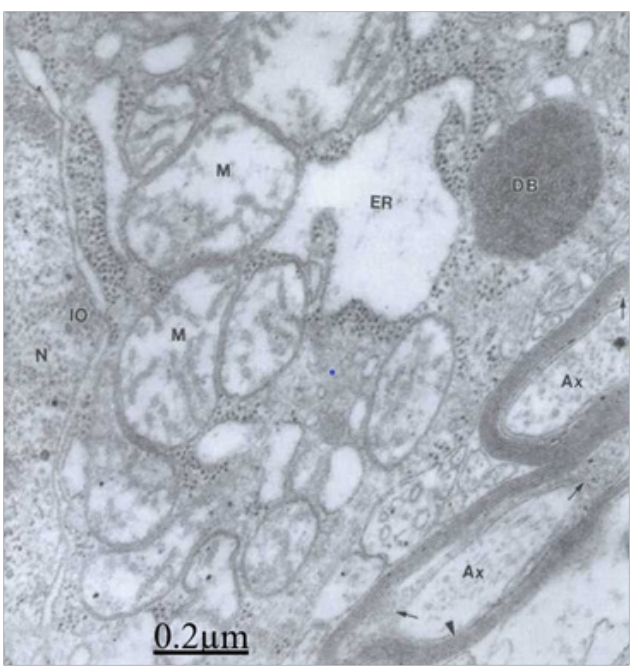

Figure 12 Brain traumas, hematoma subdural. Severely edematous oligodendrocyte showing the nucleus $(\mathrm{N})$ and lacunar enlargement of endoplasmic reticulum (ER), swollen mitochondria (M) and dense body (DB). The associated and degenerated myelinated axons show granular precipitation of axoplasm (Ax), and enlargement of periaxonal space (short arrow). The arrowhead indicates the axolemmal membrane.

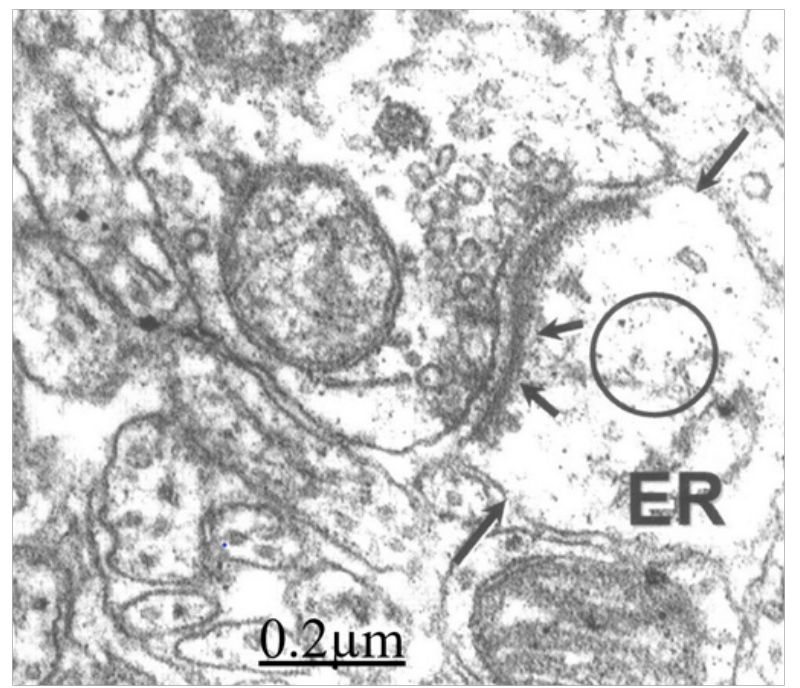

Figure 13 Brain traumas, subdural hematoma. Synaptic plasticity The preand postsynaptic membranes displayed an undulated course. The short arrows indicate the postsynaptic density. The circle shows the disrupted postsynaptic cytoskeleton. The postsynaptic endoplasmic reticulum (ER) profile appears enlarged. The long arrow indicates the fragmented limiting plasma membrane of postsynaptic ending.

\section{Severe brain trauma and synaptic disassembly}

The severely swollen brain parenchyma exhibits synaptic disassembly characterized by enlargement of synaptic cleft, wide communication with the extracellular space and detachment of perisynaptic astrocyte cytoplasm (Figure 16). ${ }^{29}$ 

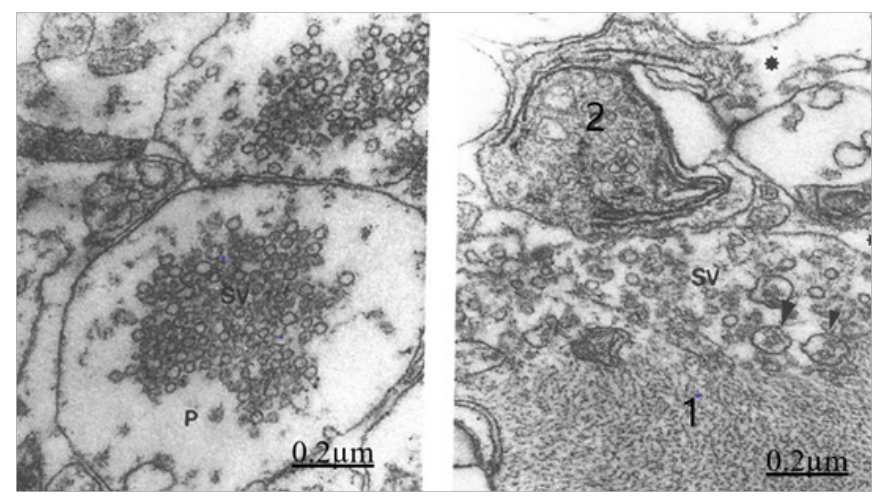

Figures 14\&15 Brain traumas, subdural hematoma. Synaptic degenerated presynaptic endings. The presynaptic ending (P) shows clustered and coalescent synaptic vesicles (SV). The right figure exhibits two degenerated presynaptic endings $(I, 2)$, small and medium size synaptic vesicles (SV). Note the filamentous hypertrophy rejecting the synaptic vesicles toward the peripheral ending zone. The asterisk indicates the enlargement of extracellular space.

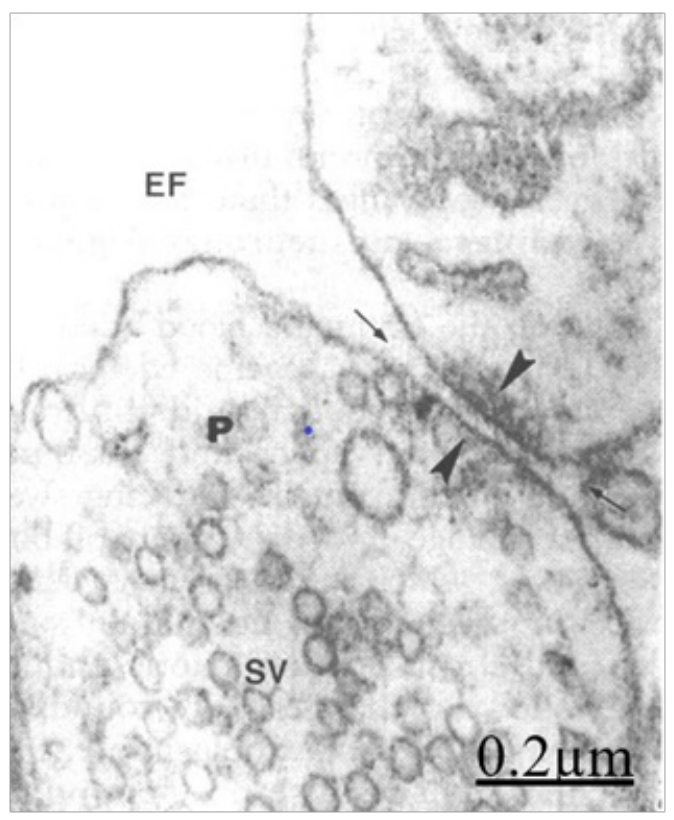

Figure 16 Brain traumas, subdural hematoma. The arrowheads indicate the synaptic disassembly featured by wide separation of pre- and postsynaptic membranes. The pre- and postsynaptic endings appear separated by a dilated synaptic cleft in continuity with the extracellular space (arrow). Note the absence of presynaptic density, the remaining thin postsynaptic density (arrowheads), and the detachment of perisynaptic astrocyte cytoplasm.

\section{Brain trauma, brain aging and degenerated myelinated axons}

At the level of neuropil we found myelinated axonal degeneration (Figures 17\&18). ${ }^{30}$ The longitudinal ultrathin sections of degenerated myelinated axons show the vacuolated myelin sheath and disruption of cytoskeletal structures (Figure 19).

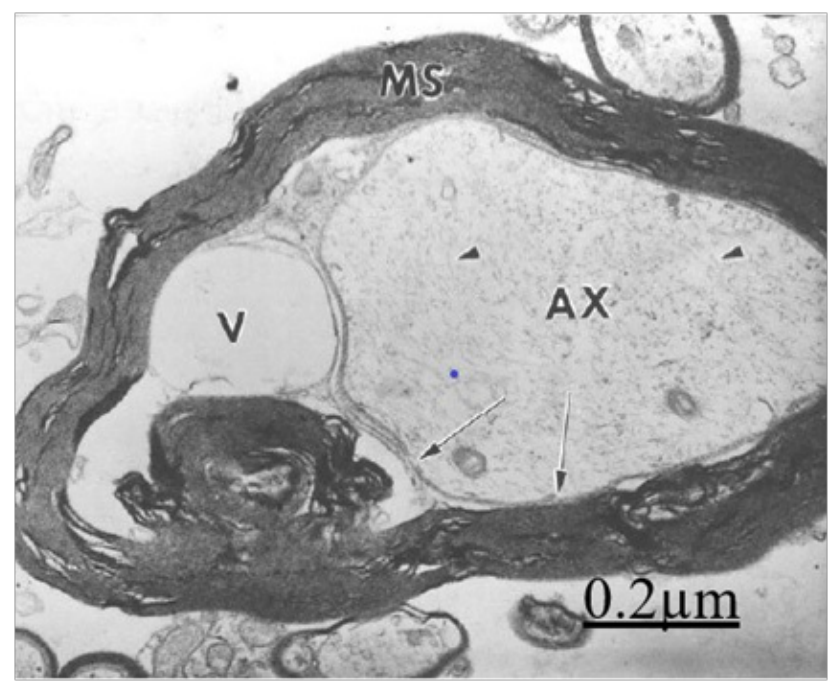

Figure 17 Brain traumas, subdural hygroma. Cross ultrathin section of a degenerated myelinated axon. The myelin sheath (MS) appeared vacuolated. The axoplasm (AX) is rejected by a large periaxonal vacuolar space $(V)$ and the formation of a myelin ovoid. The arrows point out the axolemma limiting the dilated periaxonal space.

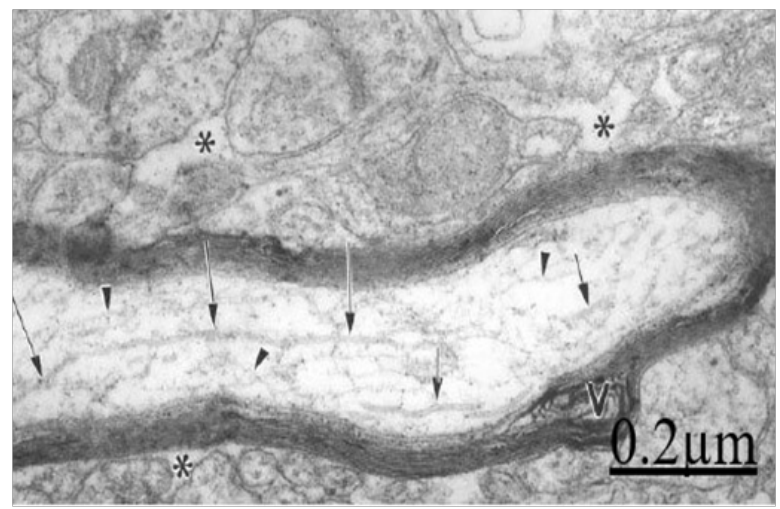

Figure 18 Brain traumas, subdural hematoma. Electron microscopy of a longitudinally section of a degenerated myelinated axon exhibiting fragmented microtubules (arrows) and microfilaments (arrowheads). Note the vacuolated myelin sheath $(\mathrm{V})$ and the enlarged neighboring extracellular space (asterisks).

\section{Brain trauma, brain aging and neurodegenerative diseases}

Clinical studies has reported a relationship between TBI and neurodegenerative disorders, such as Alzheimer's and Parkinson's diseases, as well as certain psychiatric diseases. ${ }^{31,32}$ Dall'Acqua et al. $^{33}$ have reported prefrontal cortical thickening after mild traumatic brain injury by means of a magnetic resonance imaging study.

\section{Brain trauma, endoplasmic reticulum stress and memory loss}

According to Yang et $\mathrm{al}^{34}$ and Yin et $\mathrm{al}^{35}$ endoplasmic reticulum stress has been implied in various CNS injuries, including brain ischemia, traumatic brain injury, and aging-associated neurodegeneration, such as Alzheimer's disease (AD), Huntington's 
disease (HD), Amyotrophic lateral sclerosis (ALS), and Parkinson's disease (PD). In addition, we have observed detachment of associated ribosomes and specialized arrangement of smooth endoplasmic reticulum, apparently induced by the shear forces and intensity of traumatic agent. Such ribosome detachment could partially explain the transitory memory loss of patients with severe head injuries (Figure 20). ${ }^{36}$

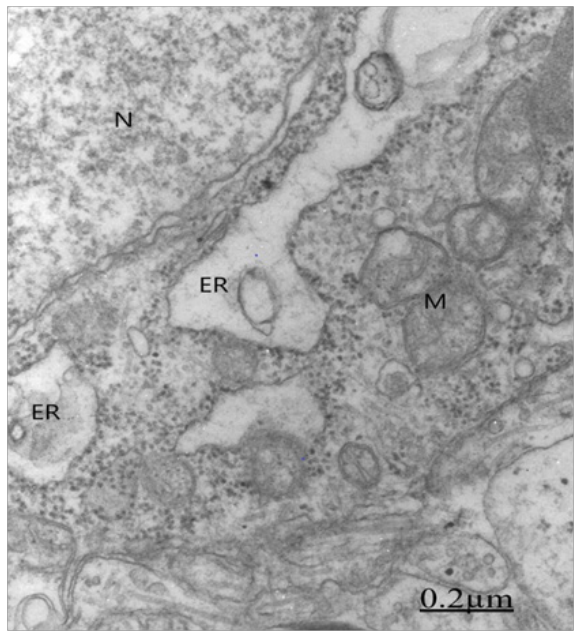

Figure I 9 Brain traumas, subdural hematoma. Non pyramidal neuron showing lacunar enlargement of rough endoplasmic reticulum (ER) and detachment of associated ribosomes (arrow).

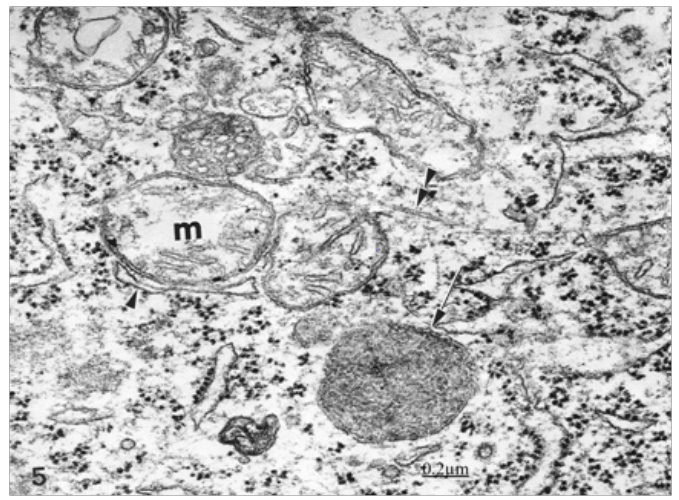

Figure 20 Brain traumas, subdural hematoma. Edematous nerve cell showing swollen mitochondria $(\mathrm{m})$, a dense degenerated mitochondria (arrow), and disrupted microtubule (double arrowhead).

Memory is fundamental to everyday life, and cognitive impairments resulting from traumatic brain injury (TBI) have devastating effects on TBI survivors. A contributing component to memory impairments caused by TBI is alteration in the neural circuits associated with memory function. ${ }^{37}$

\section{Brain trauma, brain aging and swollen and degenerated mitochondria}

We have previously reported edematous, clear and dense degenerated mitochondria in traumatic brain injuries (Figure 21). ${ }^{31,32}$ There is an interplay between mitochondrial DNA, pathophysiology of TBI, and aging. Structural and functional damage of mitochondria is currently found in traumatic brain injuries. ${ }^{37-41}$ Mitochondria contain their own DNA (mtDNA) with genomic variants that have different physiological and pathological characteristics, including susceptibility to neurodegeneration. ${ }^{42}$ Given the central role of mitochondria in the pathophysiology of neurological injury, these Authors hypothesized that its genomic variants may account for the variability in outcome following TBI. The data also suggest that the APOE pathways interact with genetically regulated mitochondrial functions in the response to acute injury, as previously reported in Alzheimer disease.

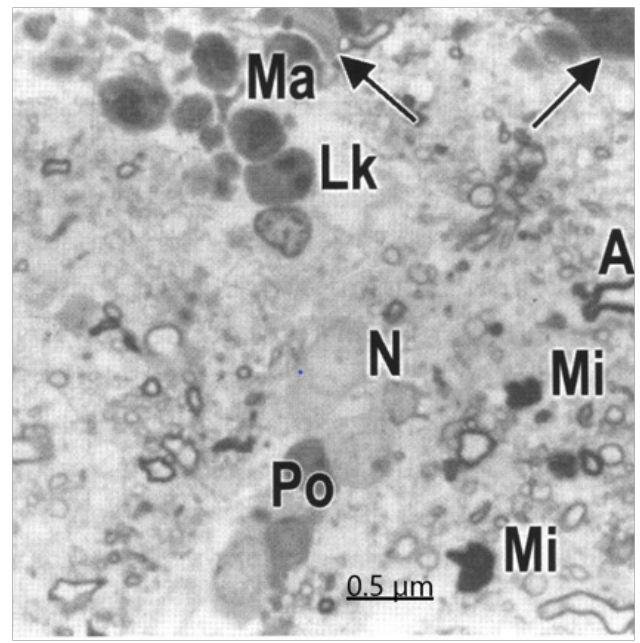

Figure 2 I Brain traumas, subdural hematoma. Inflammatory reaction featured by the presence of microglial cells (Mi), leukocytes (Lk) and macrophages (MA) invading the brain parenchyma and surrounding a nerve cell $(\mathrm{N})$. The arrows point out the dense hematogenous edema fluid.

Neuropathological studies of human traumatic brain injury (TBI) cases have described amyloid plaques acutely after a single severe TBI, and tau pathology after repeat mild TBI (mTBI). This has helped drive the hypothesis that a single moderate to severe TBI increases the risk of developing late-onset Alzheimer's disease (AD), while repeat mTBI increases the risk of developing chronic traumatic encephalopathy (CTE) ${ }^{1,43,44}$ The interplay of age at injury and aging with an injury are translationally important factors that influence behavioral performance as a quality of life metric. ${ }^{45}$

Recovery from traumatic brain injury (TBI) depends on age-atinjury and how aging with a TBI affects long-term recovery. TBI initiates pathophysiological processes that dismantle circuits in the brain. In response, reparative and restorative processes reorganize circuits to overcome the injury-induced damage. The extent of circuit dismantling and subsequent reorganization depends as much on the initial injury parameters as other contributing factors, such as genetics and age. Age-at-injury influences the way the brain is able to repair itself, as a result of developmental status, extent of cellular senescence, and injury-induced inflammation. Moreover, endocrine dysfunction can occur with TBI. Depending on the age of the individual at the time of injury, endocrine dysfunction may disrupt growth, puberty, influence social behaviors, and possibly alter the inflammatory response. In turn, activation of microglia, the brain's immune cells, after injury may continue to fuel endocrine dysfunction. With age, the immune system develops and microglia becomes primed to subsequent challenges. Sustained inflammation and microglial activation can continue for weeks to months postinjury. This prolonged inflammation can influence developmental processes, behavioral performance and age-related decline. ${ }^{46}$

According to Liu et al. ${ }^{47}$ similar to neuron-enriched gray matter areas, white matter (WM) undergoes a series of changes during the process of aging. WM malfunction can induce serious 
neurobehavioral and cognitive impairments. Thus, age-related changes in WM may contribute to the functional decline observed in the elderly. In addition, aged WM becomes more susceptible to neurological disorders, such as stroke, traumatic brain injury (TBI), and neurodegeneration. These Authors discuss how age-related WM changes influence the progression of various brain disorders, including ischemic and hemorrhagic stroke, TBI, Alzheimer's disease, and Parkinson's disease. In aged WM, the expression of GLT-1 greatly increases in multiple cellular components, resulting in more rapid and robust glutamate release and severe excitotoxicity during ischemia. Excessive oxidative stress is also likely to aggravate WM injury in the aged brain. Mitochondria are the main source of reactive oxygen species (ROS) after ischemia. Age-related mitochondrial dysfunction results in reduced ATP production and excessive oxidative stress. The functions of endogenous antioxidant systems decline with aging in parallel with the increase in mitochondrial impairments. All these changes converge to increase axonal and oligodendrocyte vulnerability in aged brains and exacerbate WM injury upon ischemia. The current state of the literature provides support for the argument that TBI can result in diminished cognitive reserve which may accelerate the normal process of cognitive decline, leading to premature aging, potentially increasing the risk of dementia. ${ }^{48}$

\section{Chronic traumatic encephalopathy}

Chronic traumatic encephalopathy (CTE) is a long-term neurodegenerative consequence of repetitive head impacts which can only be definitively diagnosed in post-mortem. Recently, the consensus neuropathological criteria for the diagnosis of CTE was published requiring the presence of the accumulation of abnormal tau in neurons and astroglia distributed around small blood vessels at the depths of cortical sulci in an irregular pattern as the mandatory features. ${ }^{49}$

The pathology of chronic traumatic encephalopathy (CTE) is increasingly well known and is characterized by a distinctive pattern of progressive brain atrophy and accumulation of hyperphosphorylated tau neurofibrillary and glial tangles, dystrophic neurites, $43 \mathrm{kDa}$ TAR DNA-binding protein (TDP-43) neuronal and glial aggregates, microvasculopathy, myelinated axonopathy, neuroinflammation, and white matter degeneration. ${ }^{43,50}$

\section{Traumatic brain injury, brain aging and demencia}

There is a long history linking traumatic brain injury (TBI) with the development of dementia. 1) Increasing severity of a single moderate-to-severe TBI increases the risk of subsequent Alzheimer's disease (AD), the most common type of dementia. 2) Repetitive, often subconcussive, mild TBIs increase the risk for chronic traumatic encephalopathy (CTE), a degenerative neuropathology. 3) TBI may be a risk factor for other neurodegenerative disorders that can be associated with dementia. 4) TBI appears to lower the age of onset of TBI-related neurocognitive syndromes, potentially adding "TBI cognitive-behavioral features". The literature further indicates several specific risk factors for TBI-associated dementia: 5) any blast or blunt physical force to the head as long as there is violent head displacement; 6) decreased cognitive and/or neuronal reserve and the related variable of older age at TBI; and 7) the presence of apolipoprotein E $\varepsilon 4$ alleles, a genetic risk factor for AD. Finally, there are neuropathological features relating TBI with neurocognitive syndromes: 8) acute TBI results in amyloid pathology and other neurodegenerative proteinopathies; 9) CTE shares features with neurodegenerative dementias; and 10) TBI results in white matter tract and neural network disruptions. ${ }^{51}$

Sayed et al. ${ }^{52}$ found that patients with dementia after traumatic brain injuries (TBI) were significantly more likely to experience depression, anxiety, irritability, and motor disorders than patients with probable Alzheimer disease. Brenowitz et al. ${ }^{53}$ examined the relationships between Alzheimer's disease, neuropathologic change (ADNC), Lewy body disease (LBD), and vascular brain injury (VBI) in two large autopsy samples. According to these Authors, high prevalence of co-occurring neuropathologies among older adults with dementia has implications for accurate diagnosis of dementia etiologies and development of disease-modifying strategies.

Research that evaluates number and severity of TBIs is needed to clarify the neuropathological links between TBI and dementia documented in other large clinical databases. ${ }^{54}$

Concussion and other traumatic brain injuries (TBIs) and Alzheimer's disease (AD) share many overlapping pathologies and possible clinical links. Such studies may demonstrate the potential for drugs to effectively and safely inhibit preprogrammed cell death as a new drug development strategy for use in the fight to defeat $A D .^{55}$

\section{Brain trauma, neuroinflammatory reaction and old brain}

Norden et al. ${ }^{56}$ emphasize the microglial priming and enhanced reactivity to secondary insult in aging, and traumatic CNS injury, and neurodegenerative disease. Microglia can develop a primed or pro-inflammatory mRNA, protein and morphological profile with aging, traumatic brain injury and neurodegenerative disease.

The light microscopy study shows the invading cells, such as macrophages and monocytes that occurred after trauma (Figure 22). ${ }^{57}$

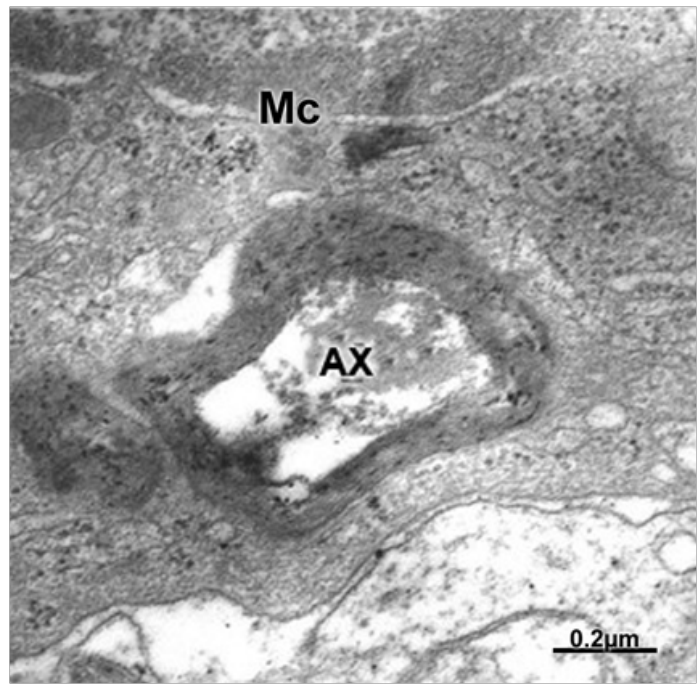

Figure 22 Brain traumas, subdural hematoma. Microglial cell (MC) showing phagocytic activity of a degenerated myelinated axon (AX).

\section{Microglial function, brain trauma and brain aging}

According to Bickford et al. ${ }^{58}$ aging leads to altered microglial function that reduces brain resiliency increasing vulnerability to neurodegenerative diseases. There is evidence that human and rodent 
microglia may become senescent. This event determines alterations in the microglia activation status, associated with a chronic inflammation phenotype and with the loss of neuroprotective functions that lead to a greater susceptibility to the neurodegenerative diseases of aging. Figure 23 illustrates a microglial cell phagocyting a degenerated myelinated axon.

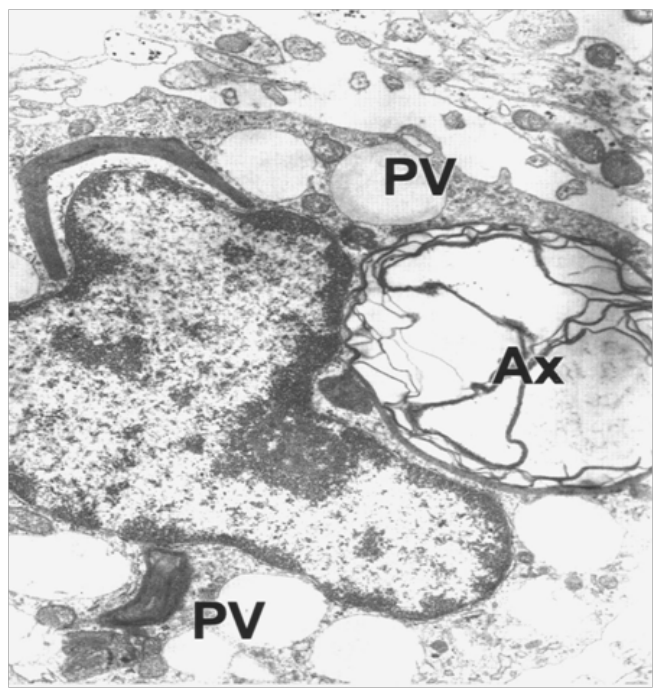

Figure 23 Brain traumas, subdural hygroma. Monocyte phagocyting a degenerated myelinated axon (AX). Note the phagocytic vacuoles (PV).

Peripherally-derived monocytes infiltrate the injured brain and contribute to chronic TBI-induced cognitive deficits in young animals. Furthermore, age was shown to amplify monocyte infiltration acutely after injury. Figure 23 shows a monocyte phagocyting a degenerated myelinated axon. Recent research with neuropathologic or biomarker evidence of Alzheimer's disease (AD) casts doubt on traumatic brain injury (TBI) as a risk factor for $\mathrm{AD} .^{54}$

\section{Conclusions}

Light and electron microscopic studies of traumatic agents on brain aging demonstrated status spongiousus of brain parenchyma, dark ischemic neuron and glial cells, degeneration of myelinated axons, inflammatory reaction, increased vesicular and vacuolar transendothelial transport, formation of transendothelial channels, closed and open interendothelial junctions, thickening and vacuolization of basement membrane, swollen and activated pericites participating in transcapillary exchange process, glio-basal dissociation, and proliferation of connective fibers. Cortical capillaries showed blood bran barrier breakdown, perivascular hemorrhages and the formation of vasogenic and cytotoxic brain edema.

Presence of swollen glycogen rich- and glycogen depleted astrocytes indicated mobilization of glycogen granules to supply ischemic neurons and ischemic and hydropic oligodendrocytes indicating the nursing role of astrocytes. The intensity of brain trauma induced degenerated myelinated axons, nerve cell death types, such as apotosis, oncosis, autophagic and necrotic nerve cell types, beaded shape and vacuolated dendrites, synaptic plasticity, synaptic degeneration and synaptic junction disassembly. The increased nerve cell death induced neurodegeneration on the aging brain. Alterations in the senescent microglia activation status, associated with a chronic inflammation phenotype and with the loss of neuroprotective functions lead to a greater susceptibility to the neurodegenerative diseases of aging.

Nerve cell organelles showed clear, dense and necrotic mitochondria, lacunar enlargement of rough endoplasmic reticulum and ribosome detachment, fragmentation of Golgi apparatus, and increased amount of lipofucsin granules in neurons and astrocytes. These findings mainly the swollen and degenerated mitochondria and the fragmentation of Golgi complex should be consider marker of nerve cell death. Clinical studies reported a relationship between TBI and neurodegenerative disorders, such as Alzheimer's Parkinson's diseases, amyotrophic lateral sclerosis as well as certain psychiatric diseases, such as chronic traumatic encephalopathy, cognitive decline, depression, anxiety, irritability, motor disorders, endocrine dysfunction, confusion and demencia. Genetics, age, inflammatory reaction and intensity of brain trauma are risk factors in the genesis of neurodegeneration. The electron microscopic findings indicate cytotoxic and vasogenic brain oedema, cell death, and neurodegenerative processes leading to neurological and mental diseases.

\section{Acknowledgement}

This review has been carried out by a subvention obtained from Biological Research Institute. Faculty of Medicine, Zulia University and Castejón Foundation.

\section{Conflicts of interest}

Author declares there are no conflicts of interest.

\section{References}

1. Gardner RC, Yaffe K. Epidemiology of mild traumatic brain injury and neurodegenerative disease. Mol Cell Neurosci. 2015;66(Pt B):75-80.

2. Adle-Biassette H, Duyckaerts C, Wasowicz M, et al. Beta AP deposition and head trauma. Neurobiol Aging. 1996;17(3):415419 .

3. Irimia A, Van Horn JD, Vespa PM. Persistent Infiltration and Impaired Response of Peripherally-Derived Monocytes. Int J Mol Sci. 2018;19(6). pii:E1616.

4. Hanif S, Abodunde O, Ali Z, Pidgeon C. Age related outcome in acute subdural haematoma following traumatic head injury. Iranian Med J. 2009;102(8):255-257.

5. Miakotnykh VS, Chursin SA, Borovkova TA, et al. Some problems of interrelations between ischemic stroke and cranial-cerebral trauma in elderly and aged patients. Adv Gerontol. 2007;20(4):8388 .

6. Castejón OJ.Electron microscopy study of capillary wall in human cerebral edema. J Neuropathol Exp Neurol. 1980;39(3):296-328.

7. Castejón OJ. Increased vesicular and vacuolar transendothelial transport in traumatic human brain oedema. A review. Folia Neuropathol. 2013;51(2):93-102.

8. Castejón OJ. Electron microscopic observations of endothe $\neg$ lial junctions in perifocal human brain edema. An interpretative study. J Submicrosc Cytol. 1985;17(1):105-114. 
9. Castejón OJ. Ultrastructural pathology of endothelial tight junctions in human brain oedema. A Review. Folia Neuropathol. (Poland). 50(2):118-129.

10. Castejón OJ. Formation of transient transendothelial channels in traumatic human brain edema. Path Res Pract. 1984;179 (1):7-12.

11. Castejón OJ. Ultrastructural alterations of human cortical capillary basement membrane in perifocal brain edema. J. Submicrosc. $J$ Submicrosc Cytol Pathol. 1988;20(3):519-536.

12. [13]. Castejón OJ. (2014) Ultrastructural alterations of human cortical capillary basement membrane in perifocal brain edema. A Review. Folia Neuropathol. 52:10-21.

13. Castejón OJ. Submicroscopic changes of cortical capillary pericytes in human perifocal brain edema. J Submicrosc Cytol. 1984;16(3):601-618.

14. Castejón OJ. Ultrastructural pathology of cortical capillary pericytes in human traumatic brain oedema. A Review. Folia Neuropathol. 2011:49(3):162-173.

15. Castejón OJ, Valero C, Díaz M. Light and electron microscopy of nerve cells in traumatic oedematous human cerebral cortex. Brain Inj. 1997;11(5):363-388.

16. Castejón OJ, Arismendi G. Nerve cell death types in the edematous human cerebral cortex. J Submicrosc Cytol Pathol. 2006;38(1):2136.

17. Castejón OJ, Arismendi G. Morphological changes of dendrites in the human edematous cerebral cortex. A transmission electron microscopic study. J Submicrosc Cytol Pathol. 2003;35(4):395413.

18. Castejón OJ. Ultrastructural morphology of dendritic spines in normal and some neuropathological conditions. A review. $J A d v$ Microsc Res. 2014;9:163-178.

19. Castejón OJ, Díaz M, Castejón HV, et al. Glycogen-rich and glycogen- depleted astrocytes in the oedematous human cerebral cortex associated with brain trauma, tumours and congenital malformations:an electron microscopy study. Brain Injury. 2002;116(2):109-132.

20. Castejón OJ. Biopathology of astrocytes in human traumatic and complicated brain injuries. Review and hypothesis. Folia Neuropathol. 2015;53(3):173-192.

21. Philbin DM, Ransom BR. Anoxia induced extracellular ion shifts in mammalian CNS white matter. In:S Federoff, HJ Bernhard, editors. Biology and Pathology of Astrocytes-Neuron Interaction. NewYork:Plenum Press; 1993. pp. 27-50.

22. Castejón OJ. Castejón HV. Astrocyte subtypes in the gray matter of injured human cerebral cortex. A transmission electron microscopic study. Brain Injury. 1999;13(4):291-304.

23. Castejón OJ. Electron microscopy of astrocyte changes and subtypes in traumatic human edematous cerebral cortex:a review. Ultrastruct Pathol. 2013;37(6):417-424.

24. Castejón OJ, Castejón HV. Oligodendroglial cell behaviour in traumatic oedematous human cerebral cortex. A light and electron microscopic study. Brain Injury. 2000;14:303-317.
25. Castejón OJ. Ultrastructural pathology of oligodendroglial cells in traumatic and hydrocephalic human brain edema:A review. Ultrastruct Pathol. 2015;39(6):359-368.

26. Castejon OJ. Synaptic plasticity in severe human traumatic brain injuries:an electron microscopic study using cortical biopsies. $J$ Adv Microsc Res. 2017;12(2):97-103.

27. Castejón OJ, Valero C, Díaz M. Synaptic degenerative changes in human traumatic brain edema. J Neurosurg Sci. 1995;39(1):47-65.

28. Castejón OJ. Electron microscopic analysis of cortical biopsies in patients with traumatic brain injuries and dysfunction of neurobehavioral system. $J$ Submicrosc Cytol Pathol. 1998;30(1):145-156.

29. Castejón OJ. Electron microscopic study of central axonal degeneration in traumatic human brain edema. J Submicrosc Cytol. 1985;17(4):703-718.

30. Castejón OJ. Structural pattern of injured mitochondria in oedematous human cerebellar cortex. Brain Injury. 2004;18(11):1107-1126.

31. Castejón OJ. The thesis of mitochondria as marker of lethal injury in the traumatic human brain edema. An electron microscopic study using cortical biopsies. Acta Microscópica. 2008;17:16-27.

32. Dall'Acqua P, Johannes S, Mica L, et al. Prefrontal Cortical Thickening after Mild Traumatic Brain Injury: A OneYear Magnetic Resonance Imaging Study. $J$ Neurotrauma. 2017;34(23):3270-3279.

33. Yang F, Luo J. Endoplasmic reticulum stress and ethanol neurotoxicity. Biomolecules. 2015;5(4):2538-2553.

34. Yin Y, Sun G, Li E, et al. RE stress and impaired autophagy flux in neuronal degeneration and brain injury. Ageing Res Rev. 2015;34:3-4.

35. Castejón OJ, Valero C, Díaz M. Light and electron microscope study of nerve cells in traumatic oedematous human cerebral cortex. Brain Injury. 1997;11(5):363-388.

36. Lifshitz J, Friberg H, Neumar RW, et al. Structural and functional damage sustained by mitochondria after traumatic brain injury in the rat:evidence for differentially sensitive populations in the cortex and hippocampus. J Cereb Blood Flow Metab. 2003;23(2):219-231.

37. Enriquez P, Bullock R. Molecular and cellular mechanisms in the pathophysiology of severe head injury. Curr Pharm Des. 2004;10(18):2131-2143.

38. Xiong Y, Shie FS, Zhang J, et al. Prevention of mitochondrial dysfunction in post-traumatic mouse brain by superoxide dismutase. J Neurochem. 2005;95(3):732-744.

39. Marmarou A, Signoretti S, Fatouros P, et al. Mitochondrial injury measured by proton magnetic resonance spectroscopy in severe head trauma patients. Acta Neurochir Suppl. 2005;95:149-151.

40. Lifshitz J, Janmey PA, McIntosh TK. Photon correlation spectroscopy of brain mitochondrial populations:application to traumatic brain injury. Exp Neurol. 2006;197(2):318-329. 
41. Bulstrode H, Nicoll JA, Hudson G, et al. Mitochondrial DNA and traumatic brain injury. Ann Neurol. 2014;75(2):186-195.

42. Daneshvar DH, Goldstein LE, Kiernan PT, et al. Posttraumatic neurodegeneration and chronic traumatic encephalopathy. Mol Cell Neurosci. 2015;66(Pt B):81-90.

43. Washington PM, Villapol S, Burns MP. Polypathology and dementia after brain trauma: Does brain injury trigger distinct neurodegenerative diseases, or should they be classified together as traumatic encephalopathy? Exp Neurol. 2016;275. Pt 3:381-388.

44. Rowe RK, Ziebell JM, Harrison JL, et al. Aging with traumatic brain injury:effects of age at injury on behavioral outcome following diffuse brain injury in rats. Dev Neurosci. 2016;38(3):195-205.

45. Ziebell JM, Rowe RK, Muccigrosso MM, et al. Aging with a traumatic brain injury:Could behavioral morbidities and endocrine symptoms be influenced by microglial priming? Brain Behav Immun. 2017;59:1-7.

46. Liu H, Yang Y, Xia Y, et al. Aging of cerebral white matter. Ageing Res Rev. 2017;34:64-76.

47. Wood RL. Accelerated cognitive aging following severe traumatic brain injury: A review. Brain Inj. 2017;31(10):1270-1278.

48. Ling H, Neal JW, Revesz T. Evolving concepts of chronic traumatic encephalopathy as a neuropathological entity. Neuropathol Appl Neurobiol. 2017;43(6):467-476.

49. McKee AC, Cairns NJ, Dickson DW, et al. TBI/CTE group Alzheimer's disease neuropathologic change, Lewy body disease, and vascular brain injury in clinic-and community-based samples. Neurobiol Aging. 2017;53:83-92.

50. Mendez MF. Prefrontal cortical thickening after mild traumatic brain injury:a one-year magnetic resonance imaging study. $J$ Neurotrauma. 34(23):3270-3279.
51. Sayed N, Culver C, Dams-O'Connor K, et al. Clinical phenotype of dementia after traumatic brain injury. $J$ Neurotrauma. 2013;30(13):1117-1122.

52. Brenowitz WD, Keene CD, Hawes SE, et al. Aging leads to altered microglial function that reduces brain resiliency increasing vulnerability to neurodegenerative diseases. Exp Gerontol. $2017 ; 94: 4-8$

53. Sugarman MA, McKee AC, Stein TD, et al. Failure to detect an association between self-reported traumatic brain injury and Alzheimer's disease neuropathology and dementia. $J$ Alzheimers Dis. 2019;57(3):667-681.

54. Becker RE, Kapogiannis D, Greig NH. Failure to detect an association between self-reported traumatic brain injury and Alzheimer's disease neuropathology and dementia. Alzheimers Dement. Alzheimers Dement. 2019;15(5):686-698.

55. Norden DM, Muccigrosso MM, Godbout JP. Microglial priming and enhanced reactivity to secondary insult in aging, and traumatic CNS injury, and neurodegenerative disease. Neuropharmacology. 2015;96(Pt A):29-41.

56. Castejón OJ. Castellano A, Arismendi GJ, et al. The inflammatory reaction in human traumatic brain edema. J. Submicrosc. $J$ Submicrosc Cytol Pathol. 2005;37(1):43-52.

57. Mecca C, Giambanco I, Donato R, et al. Microglia and aging: the role of the TREM2-DAP12 and CX3CL1-CX3CR1. Int J Mol Sci. 2018;19(1). pii: E318.

58. Chou A, Krukowski K, Morganti JM, et al. Does traumatic brain injury hold the key to the Alzheimer's disease puzzle? Alzheimers Dement. 2018;14(4):431-443. 